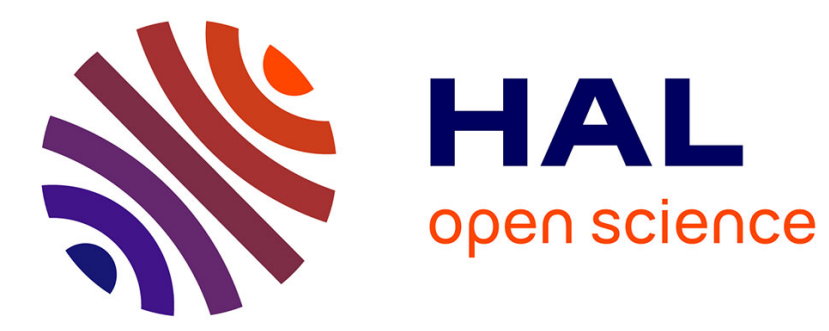

\title{
Le théâtre gallo-romain de Canouville (Seine-Maritime)
}

Jacques Le Maho, Jean Nicolle

\section{To cite this version:}

Jacques Le Maho, Jean Nicolle. Le théâtre gallo-romain de Canouville (Seine-Maritime). Gallia - Fouilles et monuments archéologiques en France métropolitaine, 1979, 37 (2), pp.237-246. 10.3406/galia.1979.1603 . hal-01934118

\section{HAL Id: hal-01934118 https://hal.science/hal-01934118}

Submitted on 3 Mar 2020

HAL is a multi-disciplinary open access archive for the deposit and dissemination of scientific research documents, whether they are published or not. The documents may come from teaching and research institutions in France or abroad, or from public or private research centers.
L'archive ouverte pluridisciplinaire HAL, est destinée au dépôt et à la diffusion de documents scientifiques de niveau recherche, publiés ou non, émanant des établissements d'enseignement et de recherche français ou étrangers, des laboratoires publics ou privés.

\section{(1) $(1) \Theta$}

Distributed under a Creative Commons Attribution - NonCommercial - NoDerivatives| 4.0 


\title{
LE THÊÂTRE GALLO-ROMAIN DE CANOUVILLE (Seine-Maritime)
}

\author{
par Jacques LE MAHO ef Jean-Marie NICOLLE
}

Canouville ${ }^{1}$ est un pelit village de seineMaritime situé sur le plateau du Pays de Caux, à $5 \mathrm{~km}$ de la mer, entre Fécamp el saintValery-en-Caux (lig. 1). Xon loin d'un cimetiere a incinération connu depuis le $x \mathrm{x}^{\mathrm{e}}$ siècle $^{2} \mathrm{y}$ a été découvert, en janvier 1977, un théàtre qui a pu faire l'objet d'une fouille partielle.

Le village actuel se trouve à $90 \mathrm{~m}$ d'altitude, sur le rebord ouest d'une vallée sèche rejoignant a Paluel le cours d'une petite rivière côtière, la Durdent. Ces pentes s'opposent a la partie ouest du terroir qui n'est qu'une plaine à perte de vue (fig. 1). On y trouve des argiles a silex dont la plus grande partie est couverte de taillis (bois de Bascourl), tandis que la surface du plateau se compose d'un dépòt de limon gras sur un sous-sol de loess quaternaire.

I'église paroissiale est siluée à 500 m de la pente, pris d'un carrefour en étoile. Cette zone est peu bâtie. Le plus gros de l'agglomération se rassemble de part et d'autre du carrefour sur deux chemins latéraux qui suivent un tracé nord-sud, la liue-du-Hamel et la Rue-du-Bas. C'est dans un labour situé immédiatement

1 Canton de Cany-Barville, arrondissement du II:IVIe.

2 Abbe Coc:nla, La seine-Infiriente hislorique et archologgique, Paris, 1866, p. t5is; Bull. de la sioc. Norm. d'Et. Préhist., 1. 24, 1919-1921. a l'ouest du carrefour qu'ont cité irlentifiés les vestiges du théatre ${ }^{3}$.

Ice théatre se signalait avant la fouille par un renflement du sol, haut de 2,50 m, qui passait dans la tradition locale pour ètre l'emplacement d'une enceinte fortiliée du Ioyen-ige. Les premières recherches effectuées a titre de sauvetage en janvier 1977 ont révélé qu'il s'agissait en réalité d'un théàtre construil sur un monticule de terres rapportées. Sion hémicycle regarde le plein nord el ses remblais sont maintenus par un mur de silex el de luf qui décrit un arc de cercle outrepassé dont le diametre maximal est de 75) $\mathrm{m}$ (fig. 2). Au nord ont été reconnus les vestigres d'un mur de scène d'un développement de $60 \mathrm{~m}$, soit une longueur égale à la distance qui sépare ce mur de la summa caveat.

lne fois loralisé par des sondages a la tarière, le mur d'enceinte a été mis au jour sur différentes seclions de son tracé (fig. 3). Ses fondations se composent d'un amas de pierres sèches tassées dans une tranchée verticale de mêmes dimensions que le mur, et supportant un radier maconné en silex et mortier sableux large d'un metre. Ie mur lui-mème débule à $40 \mathrm{~cm}$ au-dessus du nivean

3 Caulastre $A: 45$, coord. lamberl $235,70-475,1 x$.

4 Celle disposilion correspond aux normes exposées par litrure. 


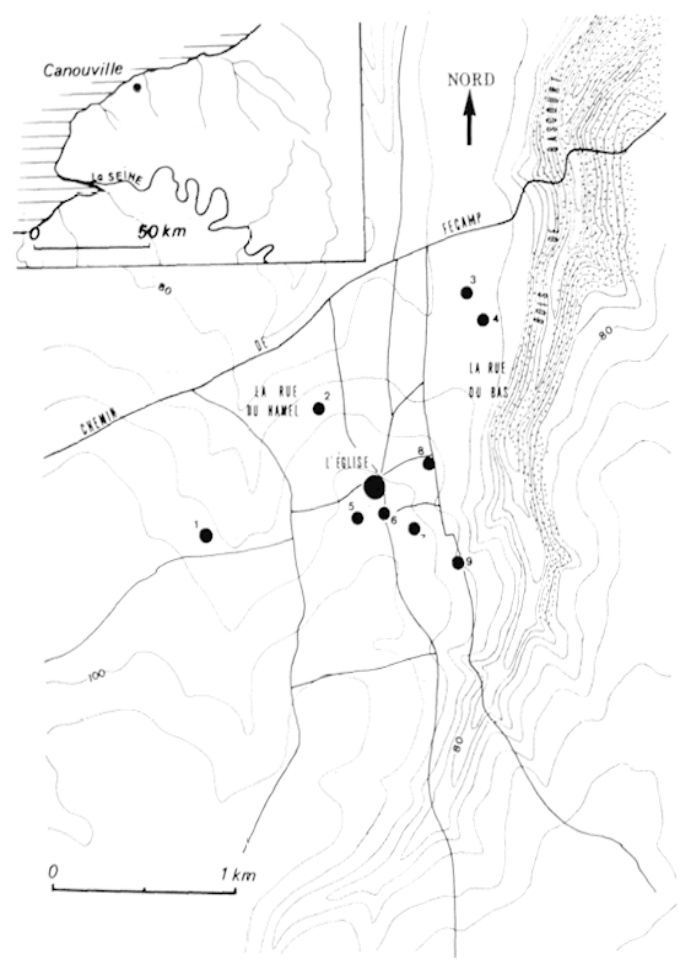

1 Canouville, plan de situation. Ies mumeros renvoient a l'inventaire de la p. 2.5 ; au centre, le theatre. II semble (que l'agglomeration gallo-romaine se soil fixé sur la limile du bois qui recouvait, dans l'anliefuiti, les pentres argilauses du vallon.

de circulalion sur un second banc de maconnerie romportant des joints tracés au fer (lig. 4 . Epais de 90 $\mathrm{cm}$, il est fait d'un blocage de silex revêtu d'un petil appareil de tuf. Iucune brique de chaînage n'a été retrouvée dans ses éboulis. Le sondage 6 contenait un grand pan de parement de tuf lombé d'une seule piece au moment de la ruine du théâtre. Lal masse de pierres s'est abatlue sur place sans que ses débris se dispersent el a donc permis d'évaluer la hauleur minimale du batiment qui était, avec 49 moellons, de $6 \mathrm{~m}$ au-dessus du sol de rirculation. Son examen donne a penser d'autre part que le mur était lisse et sans décrochement sur loule la hauteur considérée (lig. 5) et 8).

Dans le mème sondage sont apparues les bases de deux contreforts appuyés perpendiculairement au parement externe du mur. leur longueur est de 1, (bi) m el leur esparement d'aulant. re qui pourrait indiquer l'emploi d'une mesure de i) pieds. Ils ont été éleves en même lemps que le mur du théalre car leur maconnerie est commune avec celui-ci au moins jusqu'au second massif de silex (fig. 6). Mais la fouille a montré qu'ils reposaient sous un dépòt de quatre couches de travail alternant avec des remblais de limon et recouvertes en dernier lieu par de menus débris de tuf tombés sur le sol au cours de la construction du théatre. Ces couches qui étaient en parfaite roncordance avec l'empilement des remblais accumulés à l'intérieur du bâtiment étaient scellées par la nappe de terre fine sur laquelle s'est abattu le mur du théatre lors de sa chule. Les contreforts n'existaient donc pas en élévation lors de la ruine du bâtiment, et il faut conclure soit à une reprise de la construclion après arasement parliel du bâtiment - mais la stratigraphie n'indique aucun épisode de démolition ; soit plutiot à un repentir, cette seconde hypothese étant rendue assez vraisemblable par l'absence de lout contrefort dans les autres sections dégagées par la fouille $(22 \mathrm{~m}$ de mur ont été mis au jour dans le seul sondage 6).

Au nord-est, une porte large de $4 \mathrm{~m}$ s'ourre dans l'enceinle au voisinage du mur de scine. donnant accis a l'orchestre et aux rangs inférieurs de la cavea. Elle n'a conservé ni seuil, ni piédroits. L'entrée qui devait lui faire face, a l'angle opposé du bâtiment. a disparu lors de la construction de la route 1). 69 (fig. 2).

Le mur de scine (fig. 7) n'est conservé qu'au niveau des fondations, sur une longueur de 15) $m$ a partir de l'angle nord-est du bâtiment. le reste a été entraîné par le ruissellement. des eaux superficielles venues de la cacea qui ont en mime temps érodé le sol antique au voisinage du centre de la scine. L'ouvrage présente la mème épaisscur que le mur d'enceinte de la carea $(90 \mathrm{~cm})$, et il n'a pas non plus livré de décor. La fouille n'a pas révélé d'avant-scine ni de postscaenium.

L'orchestre el l'agger ont pu faire l'objel d'un relevé straligraphique complet gràce au creusement d'une tranchée axiale nord-sud (tig. 9). La piste de l'orrhestre, vraisemblablement circulaire ou subcirculaire ${ }^{5}$, occupe la

5) Eat raison de laare oultepassie forme far lo mur dencente. Cf. les plans publies par A. Ghevisk, Mranuel d'Srchéologie gallo-romaine, 3" partic, 2, 195x, prissim. 


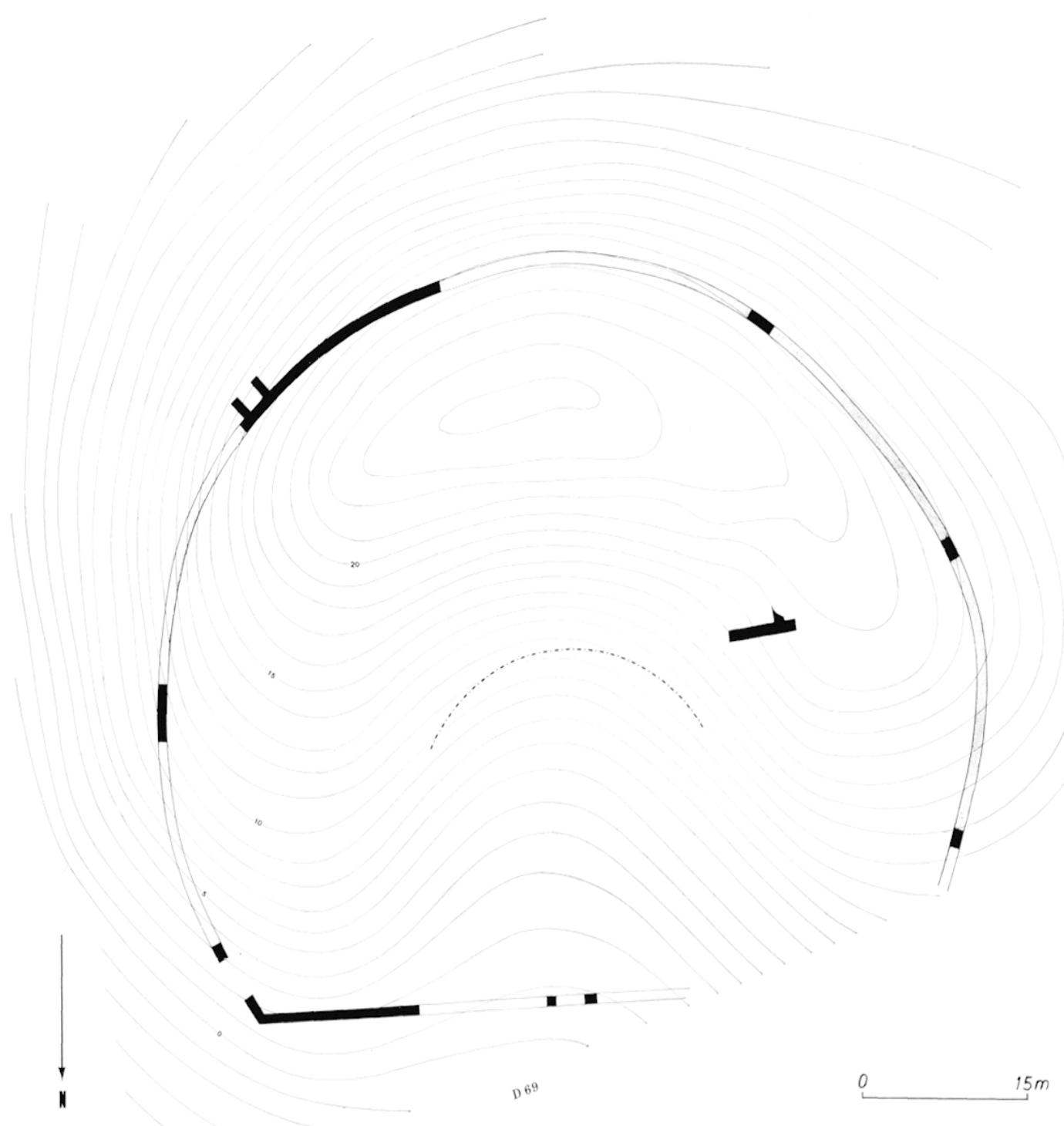

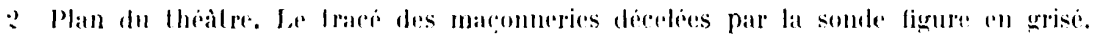

mème longueur de terrain que la cavea, soit, 30 m. L'examen de la coupe montre d'autre part, qu'elle n'a pass élé aménagée par surcreusement. si son profil est, légrèrement concave, elle se trouve au même niveau que le sol d'alentour. Sa surface se confond avec la limite supérieure du mort-terrain que recouvrent sans transilion visible les limons déposés apreśl'abandon du bàtiment. L'absence de lrace de revetement a de quoi surprendre et pourrail laisser aroire a lexistence d'une estrade de planches soulenue par un assemblage de poutres enfoncées dans le sol. Mais il n'est pas possible d'affirmer que les quatre enfoncements visibles dans le sol de la piste correspondent bien a des trous de poteaus (lig. 9 J)-E).

L'agger est fait d'une accumulation de lerres rapportées ou alternent des limons humileres. de couleur grise ou noire, et des limons argileux orangess auxquels sont associes des dépots de ler de pereolation fig. 9 (i-1)). ()n distingue essentiellement deux couches de limon argileux succédant a l'amorce d'un premier dépot de 
même nalure qui semble disparailre dans une fosse restée ouverte au moment de lat construrtion du theitre. Cetle ravile qu'il ña pas éto posibible dexplorer finissait aux abords du mur de précinclion. en B. L.es deux autres courhes argileuses sétendent sur toute la surface de l'agger romme l'ont confirmé le sondage 10 el les excavations ouvertes en divers points du bàtiment par le service des Ponts el Chansseses au cours du mois de janvier 1977. Cetle concordance stratigraphique semble indiquer que les constructeurs du théatre ont cherché a faire alterner de facon aussi réguliore que possible les couches meubles el les couches fermes dans le but de limiler les risques de lassements diflérentiels. Ia disposition des remblats est diflérente de celle des ouvrages militaires, ceux-ri élant généralement composes de multiples charges deversées l'une sur l'aulre sans lriage próalable des materiaux, comme lorsque lon remblaie une fosse.

La troisiome rouche de limon argileux marque la limile supérieure de l'agger el doil correspondre à peu pres alu niveau antique de la carea. C'est sur relle mème couche que reposent dans le sondage 10 les fondalions d'un mur de silex appartenant a l’infrast ructure des gradins". si l'on sen lient ì re profil. la cavea présente une pente de $x$ degrés par rapport a l'horizontale, inclinaison très douce. mais normale pour un thealrum exaggeratum. Il reste a préciser qu'il s'agit de la pente des remblais porteurs el non de celle, impossible a préciser, des gradins eux-meimes. De plus, la carea est incomplete dans la mesure ou ses parties hautes se sont ébouleses vers lextérieur apres la chute du mur de soutenement. Plus lard, les labours ont eu pour effel d'adourir encore le relief du monticule el d'en faire reculer la crible vers le centre du baliment. Si lon prolonge par une ligne fidive la pente de l'agger, rette ligne alleint la hauteur de 1 man contact du mur de soutenement. L colevation minimale du mur bant de 6 m. on peut penser que la maçonnerie dépassail d'au moins 2 m le plateau de la summa crenea.

(i Cif. ci-dessoms, p. :2.:.

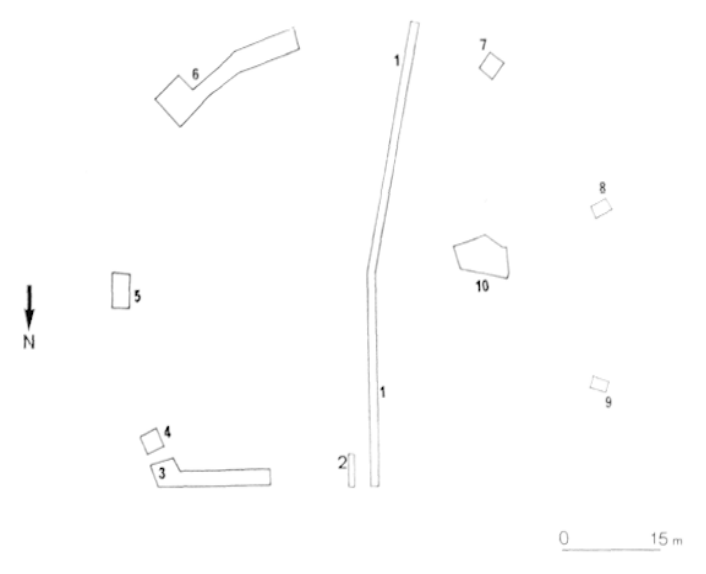

3 Pran du thralde. Numbolation dos somdages.

Au bord de l'orehestre, le remblai antique esl creusi d'une pelite dépression large de 1 m qui se présente en coupe sous la forme d'une lentille de remblais hétérogines reposanl sur une mince couche de sable de construction (lig. 9 1) . S'il peut sagir à la rigueur de la fondation d'un mur dont les superstructures auraient disparu, on concoit loutefois difficilement que relte tranchée corresponde a un podium de protection comme il en existe dans les amphitheatres. Iu lieu d'ilre suspendu au-dessus de la piste, ce que l'on attendrail en pareil cas, le remblai desend en elled tres doucement jusqu'au sol de l'orehestre.

Lat stratigraphie de l'agger présente une autre anomalie dans le secteur de la summa carea. la seconde nappe de limon argileus, qui marque semble-t-il un premier temps dam. l'édificalion du plan incliné de l'agger, retombe vers le sol a i m du mur d'enceinte au lieu de monter jusqu'au contact mème de la maconnerie. L'espace libre ainsi réservé entre l'ayger el le mur est rempli de limons gris déposés en fines pellicules horizontales qui donnent a re remblai un aspect feuilleté (fig. 9. A-C. W'autre part, l'extrémité des couches de l'agger el de la poche de limons est sectionne comme si les macons avaient a deux reprises reballe les versants du monticule avant de proceder au monlage du mur. L'ensemble de la séquence stratigraphique est donc susceptible de deux interprélalions.

A la partie enfouie de l'agger peut rorrespondre un premier théatre de terre et de bois 


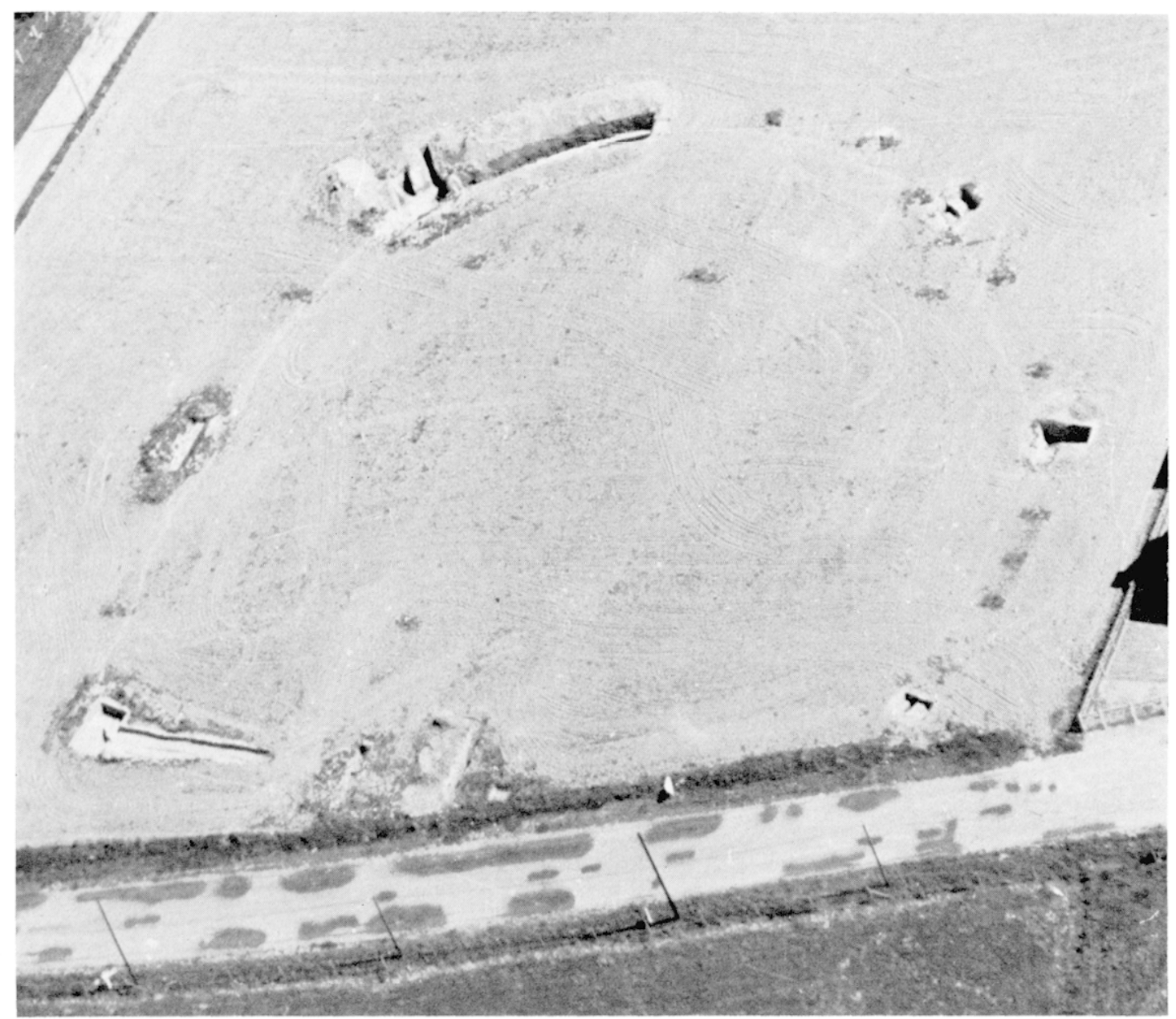

4 Vie arienne du thatre, prise du nord-ourst.

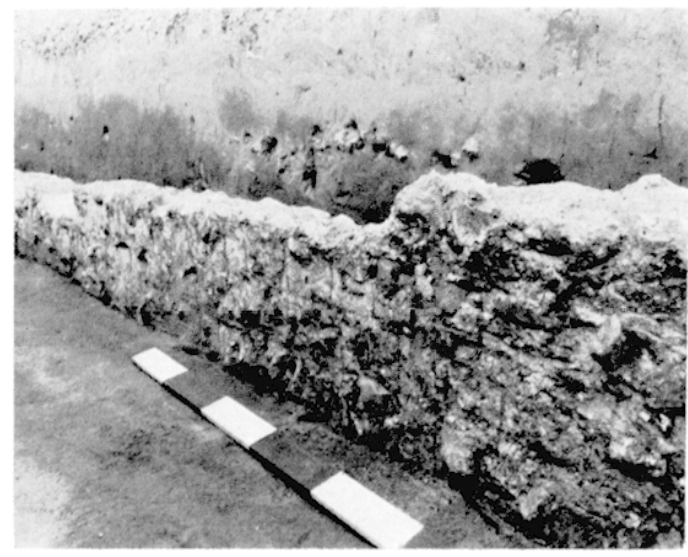

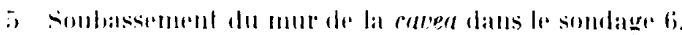

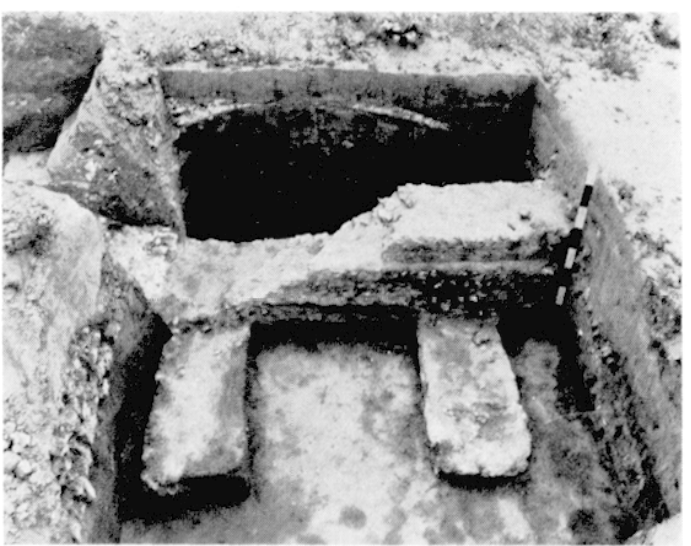

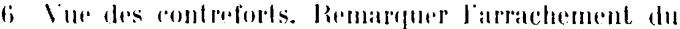
cont refort de droite sur te mur denceinte et la presenere

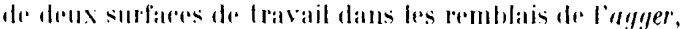
at linrriere-platl. 


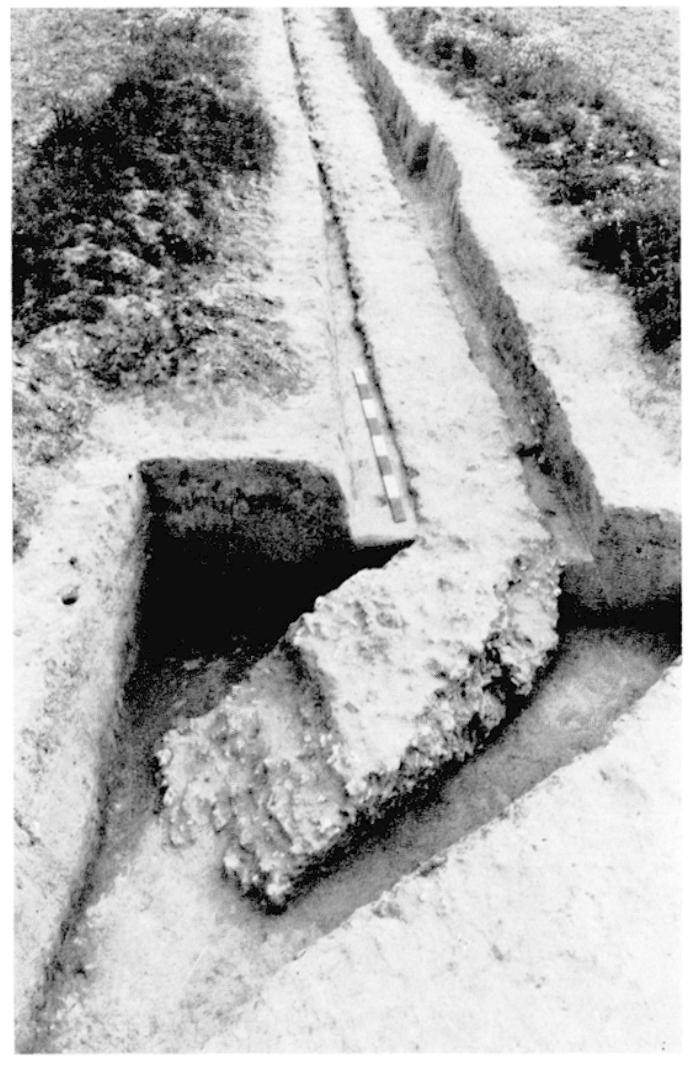

7 lev mur de scine at sa liaison alrec le mur de lat carcert.

que lion aurait dans un serond lemps augmente d'un nouvel apport de remblai el doté d'une enceinte de pierre. Cette hypothise serait corrohorée d'une certaine maniere par la présence d'un Irou de poleau sous la rrêle de l'agger (fig. 9 Ci. La base du poleau se présente comme un rylindre de terre argileuse de :30 a 40 cm de diamilre, el les limons se sont moulés autour de la piece de bois dans ses parties haules. Ie remplissage du fond ne peul provenir que de la couche d'arqile située au sommel du remblai, d'où l'on peut conclure que le potean traversail loule l'épasseur de lerre.

Rien nondique rependant que les travaux de masonnerie el de terassement ne furent pas exécutés coup sur roup. Il est possible que les conslructeurs aient arreté dans un premier lemps le plan incliné de l'agger a quelque distance du mur dans le but de limiter la pression des remblais sur la maconnerie. Une telle répartition des rharges arait en effet pour conséquence de déplacer vers l'intérieur de l'agger le point d'applicalion des poussées, le mur n'ayant qu'à supporter le petil volume de terre utilisé pour combler le vide subsistant.

S'il parait acquis de toute manière que l'agger comporte bien deux phases de construction, le tris faible éart chronologique constalé entre le mobilier recueilli sous les premiores courhes de l'agger el celui trouvé a l'extérieur des murs ${ }^{7}$ tend a faire pencher en derniere analyse pour une seule campagne de travaux. (On peut dans le même sens interpréter les coupures visibles aux abords du mur comme des reprises effectuées sur le coup pour dégager aux maggons l'espace nécessaire.

Je sommet de l'agger est traversé par trois fosses en forme dauge recoupant le dépòt limoneux qui s’était développé sur la pente de la cavea apress l'abandon du théâtre (figr. 9 B-I) . Une autre excavation d'origine récente s'ourre en avant du mur de scine ${ }^{8}$.

Le remblai antique ne portant aucune trace de revetement en dur, il faut supposer que les gradins consistaient en un assemblage de pieces de bois reposant soit sur des poleaux enfoncés dans la masse de l'agger (figr. 9 C), soit sur des murs radiaux analogues a celui dont les fondations formées de deux lits de raie tendre ont élé retrouvées sur le versant ouest de la cavea (tim. 2, sondage 10). Celle roison s'arrête net a $f ;$ m de l'orchestre el se urefle a son extrémité opposée sur un autre mur qui semble amorcer une courbe concentrique a l'enceinte du théâtre, mais dont seule une fouille plus approfondie permettrait de définir à coup sûr le tracé.

\section{Cihronologie relative el dalation.}

Il n'a élé reconnu aucune substruction sous le théatre. Et si les remblais de l'agger conliennent bien quelques tessons rapportés, essentiellement dans ses couches humifères, le mince dépoil de limon noiràtre qui marque

7 cif. ri-dessous, p. 2.13.

$x$ son remblai a liver des moremand de luiles angevines a talom pincé et un fond de jalte an gress do

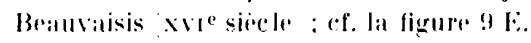


sous l'agger le niveau du sol primitif n'est sali ni de charbons de bois. ni de déchets ménagers. Il n'a livré que 13 lessons dont 2 du type de la Tine. I rette premiere occupation tris dilfuse pourail correspondre le rreusement de la fosse situé au nord du point lo idf. ri-dessus:

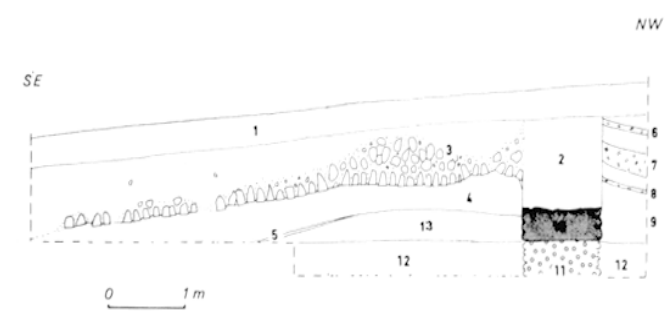

8 coupe de l'aboulis du mur de la carea sondage 6 . fonde dans le mort-lerrain le sur un massif de pierres seches 11 , le mur lo soulient wne masse de lerres rapporties allernant avec des courbes de lavail de 6 a 9 inclus . A lexterieur du hàtiment, les niveaux de construclion la remblai el a morlier sont recouverts par unt couche d'occupation t qui est scellew par l'bhoulis du mur 3 . Fin 2 , lranchea de recuperation afleurant sous la couchu arable 1.

La stratigraphie n'indique qu'une seule phase de construction pour l'ensemble des maçonneries (fig. 8, couches i) et 13 ), en dépit de la possible reprise au niveau des contreforts. I l'extérieur du mur de la canea, les aires de gàchage de mortier sont recouvertes d'un dépòt, de $20 \mathrm{~cm}$ de terre charbonneuse contenant des lessons de poterie el des déchets ménagers (fig. 8 , couche 4). Celte couche est scellée par l'éboulis du mur qui semble clore définitivement l'histoire du batiment, aucun sondage n'ayant du moins livré la preuve d'un aménagement ou d'une réoccupation des ruines, si ce n'est la découverte d'un vase mérovingien au sein d'une couche de limon rapportée sur les éboulis du mur ouest ${ }^{9}$. Dans un dernier lemps: la sourhe du mur d'enceinte a ele altaquée en plusieurs points par des récupéra-

9 Vase noir à carene, haul de lox mm, porlant un decor a la moletle sur la partie superieure de la panse; environs de la secondr moilie du va siecle idessin all dentre de recherehes darchologre medievale. Iniversite de laten. Ce vase blait isole; mais la proximiti de l'oglise suggere une destination funeraire. leurs de malériaux qui n'ont laissé que la trace du mur en nécratif, tranchée verticale comblée par du mortier désagrégé et de menus éclats de pierre (lig. 8 , couche 2 ).

la date de construction du théatre est, comprise entre deux séries de fossiles directeurs : ceux contenus dans les remblais de l'agger ou dans le niveau d'humus primitif d'une part (fig. 8. nos fi a 9). ceux qui apparliennent à la rouche d'occupation extérieure au mur dautre part $\left(n^{0} 4\right)$.

l.e premier lot contient un as de Iomitien ${ }^{10}$ recueilli a quelques centimelres du mur dans une couche limoneuse de l'agger, ainsi qu'un petit tesson de Drag. 37 portant un décor floral ef une estampille rétrograde . II.... datable du milieu ou de la seconde moilié du II $^{\mathrm{e}}$ siectell. Ce tesson qui se trouvait dans la couche végétale ancienne, sous le remblai de la media carea, fournit un terminus post quem pour la construclion du théâtre.

le deuxime lot comprend t6 tessons de poterie sigillée dont la majorité (38 framents) appartiennent à la seconde moitié du ${ }^{\mathrm{e}}$ siecle, le reste élant composé de tessons résiduels du ${ }_{1}^{\text {er }}$ sièrle et de formes non identifiées des ateliers de la Gaule centrale ${ }^{12}$. I'homogénéité de ce lot se confirme avec la céramique métallescente - 10) lessons ${ }^{13} \ldots$, avec la poterie commune ${ }^{14}$, avec enfin les monnaies. Elles couvrent une période qui va de Nerva à Hadrien, mais leur degré d'usure est tel que

11) Cohen 125, 1:2x, 131, 133 on 134, usure faible. Nous devons lidentification des monnates à l'amahilite de. Pierre-Henri Milard.

11 Il nous faut remercier Claude I.emaîlere qui a bien voulu examiner à notre demande lensemble du materiel ceramique de Camomille.

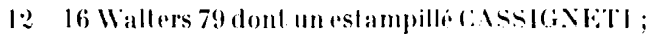

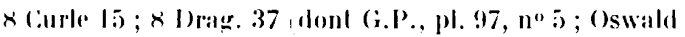
2.49, sect. 15x; Dechelelle 1105; 1 l)rag. 42 ? : I Jrag. 27 o1 35/36; I Jillerling 9 on l) rag. 24/25; 1 J)rag. 15/17:?

13 Vases du lype ciose 191; 2 fragments diun goberet noir brillant portant des filets el des points de barbotine, proches du type becheledte 72.

1.t Deux formes se degagent : un groupe de tessons apparentes alux gohelets mitallescents cose 191; plusieurs fragments dovoiddes rappelant des rases fabriques a Jisienx dans la seconde moitie du $\|^{\circ}$ siecle fonille li. Iemailer. 


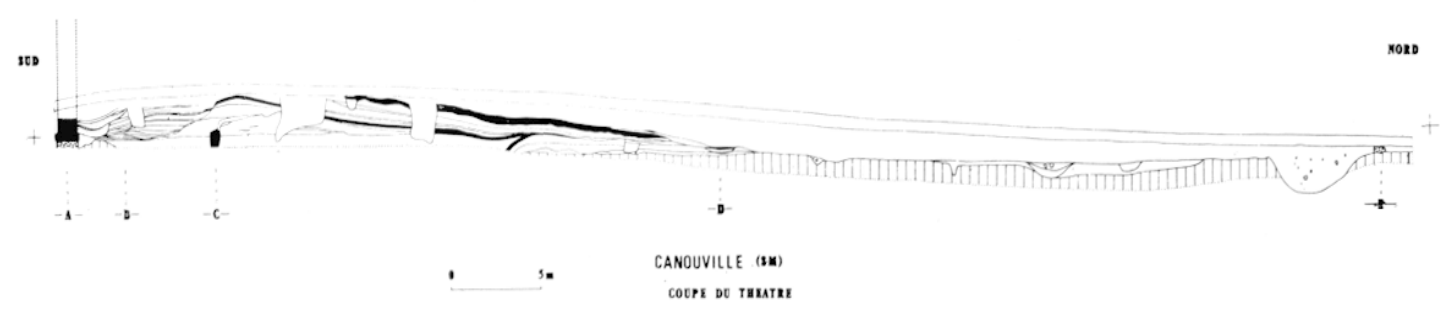

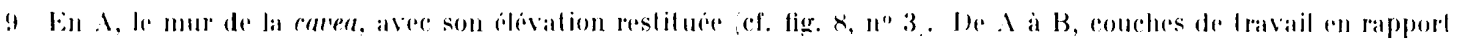

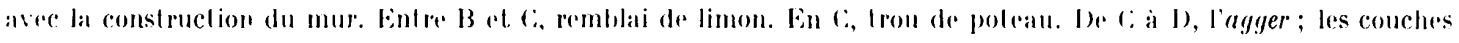
sombres sont celles qui cont iennent une forte proportion d'arpile. En l), pelile fosse conlenant du sable de construction.

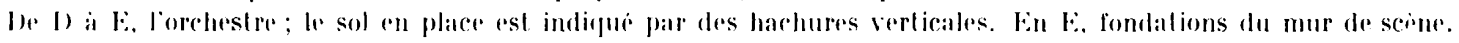

rertaines n'ont dù être perdues que dans la seconde moitié du I $^{\mathrm{e}}$ siercle $\mathrm{e}^{15}$.

Il faut donc placer à peu pris entre loo el Ist la construction du théatre. sia durée d'utilisation fut vaisemblablement tres courte. En effel. la courhe d'occupation scellée par l'éboulis. du mur n'a livré aurun tesson postérieur au ${ }_{I}{ }^{\mathrm{e}}$ sierles, absence qui a peu de chances d'etre fortuile an regard de la quantité lotale de tessons recueillis (environ 1600) \% Le plus récent est un fragment de poterie métallescente a décor de barbotine, all ribuable au dernier quart du ${ }_{1} \mathrm{e}$ siercle.

Le plan du théatre de Canouville ne présente aurune caractéristique qui puisse surprendre. Aver un diamolre de 75 m. une carea faite de lerres rapportées, des portes lalérales au niveau de l'orchestre, une piste rirculaire, il correspond aux normes les plus courantes des petits théâtres ruraux de (iaule etudiés par (i. C. Picard ${ }^{16}$. Nais sa forme de demicercle outrepassé oblige a reconsidérer une nouvelle fois le probleme de la destination de ce type de batiment qu'on a pris l'habitude de classer depuis A. Grenier dans la catégorie des "théàtres-amphithéàtres". Pour (i. C. Picard. si d'aulhentiques amphithéàtres ont bien été ulilisés romme théatres, on a peut-être trop facilement posé le schéma inverse dans le ras des théatres ruraux batis autour d'une grande piste circulaire, car la démonstration

l5 Norva colen 4, 7 ou 10, usure asser forte; Trajan Cohen 24l, usure faible ; Trajan Dupondius, usure forte : Hadrien cohen 753, 756 ou 756 , usure moyenne; Hadrien cohen 135, usure assez forte.

16 Les thidres ruraus de cianle, dans Revue. archéologique, 1970 , p. 1 $\times 5-192$. est rarement faile que celle piste ait été ronçue pour servir d'arine. Eille n'est pas toujours surcreusée el le prodium est le plus souvent inexistant, de telle sorte que les gradins descendent dans un grand nombre de cas jusquau niveau de l'orchestre. Or l'examen de la straligraphie montre qu'il en élail. de mème a Canouville. Que res dispositions aient été voulues pour des spectacles chorégraphiques. comme le sugreire G. C. Pirard. ou que nous soyons en présence d'une arene Iris rudimentaire comme à Ribemont-surIncre ${ }^{17}$. il parail raisonnable de préférer provisoirement pour Canouville le terme générique de "theatre" à celui, trop précis, de "théatre-amphithéâtre".

L'absence de briques dans les maçonneries est anormale pour une construction de lat seconde moilié du r $^{\mathrm{e}}$ siecle. Il se dégage enfin ef surtout de l'ensemble des structures mises au jour une impression d'extreme rusticité. I.e seul élément de décor recueilli dans la fouille est un fragment de base de colonne Irouvé en remploi dans les remblais de l'agger ${ }^{18}$, et dont on ne peut assurer qu'il provienne bien du chantier du théatre.

La durée tris éphémère du bâtiment (o) ans au maximum) n'a rien d'étonnant si l'on se souvient que la plupart des théatres ruraux de Gaule n'ont pas survécu à la crise du ${ }_{\text {III }}{ }^{\mathbf{e}}$ siocle. Sa destruction que l'on peut situer

17 J.-I. Cisno(x, le théatre gallo-romain de Ribemont-sur-Ancre: Essai de synthese, dans Cahiers archéologiques de Picardie, 1975, p. 49.

le Tore épais de 6 em, laillé dans un calcairte gresenx; la base complete avait un diamele d'environ in $\mathrm{cm}$. 
vers la fin du II $^{\mathrm{e}}$ sircle est contemporaine. de lincendie des deux villas de Colleville. a $12 \mathrm{~km}$ de Canouville ${ }^{19}$. On a eté tenté de mettre en rapport les niveaux de destruction de Colleville aver les lroubles politiques qui se produisirent en Gaule sous les regnes de Commode et de septime sévere ${ }^{20}$. Sagrissant cette fois-ci d'un édifice de aractere public. et qui n'a pas été remis en élal, l'éventualité d'une lin arcidentelle est d'aulant plus improbable.

\section{* *}

En altendant que des fouilles viennent préciser la nature des baliments qui entourent le théatre, les découvertes fortuites el la prospection au sol donnent une idée approximalive de l'élendue du site gallo-romain (fig. 1). Ln cimetière à incinération a livré des objets du ${ }_{11}{ }^{\mathrm{e}}$ siecle a $300 \mathrm{~m}$ au sud-est du théal re ${ }^{21}$. Vers le sud fut trouvée en 1848 une sépulture faite d'une urne en plomb déposée dans un dolium ${ }^{22}$. Enfin, dans les lerres du village, neuf emplacements de substructions ont été mis en evidence par les travaux agricoles ${ }^{23}$ :

1. Lieu-dil, La .Mi-lais, à 800 m à l'ouesl de l'église, parcelle B 70.

2. Parcelle ZB:3.

3. Lieu-dil Le Bâlimar, parcelles A 153), 154, 15).

4. I.ieu-rlil Le Bâlimar, à ?(n) m all sud-est, de la croisée des chemins, parcelles 11 199 et 1151 .

5. Lieu-dit Le Vienx-Châlean, parcelle ZB 9, entre la molte rasée au $\mathrm{xIx}^{\mathrm{e}}$ siecle el le théâtre.

6. Parcelle \% $\%$ 9. entre le théatre et l'ancienne briqueterie.

19 13. Soltudasa, La rilla gallu-romaine du PelitMoulin à Collerille (seine-Marilime), dans firmum, 3, p. 33-34.

20 Ibidem.

21 Jrag. 43 che\% Ine Fontaine, as d'Antonin (Bull. de la Soc. Norm. d Él. Préhist., 1. 21, 1919-1921. 22 Abbé Cocher, loc. cit.

23 la plupart des renseignements qui suivent nous ont ite communiques par M. Ponlel ; qu'il rll soit ici remercie.
7. Lieu-dit La Briquelerie, parcelles A 69 et 170 , cimetiere à incinération.

8. I ieu-dit La Rue-du-Bas, à l'ouest du chemin.

9. De part et d'autre du chemin de Clasville.

I.es sites 4 et 8 sont riches en reliefs alimentaires el pourraient correspondre à des habitats. A l'exception des sites 1 et 2 , les implantations gallo-romaines sont toutes situées a l'est du théatre. Eilles présentent une nette lendance à s'échelonner dans le sens nord-sud, paralliblement au vallon, et l'on peut en effet ronstater que les lahours situés immédiatement a l'ouest de cetle ligne sont exempls de traces d'occupation gallo-romaine. Il paraîl des lors vaisemblable que l'agglomération ait été faite d'un ensemble de batiments s'érrenant sur la lisiore du bois qui recouvrait les pentes aroileuses du vallon. formant, ainsi une file de constructions denviron 1 j)(0) $m$ de long. Les sépultures noccupent pas lout a fait la position excentrée qu'on est en droit d'altendre d'une nécropole; ce peut être l'indice d'une certaine progression de la surface bàtie.

I.es tessons de céramique recueillis dans les terres de Canouville vont de l'époque claudienne au $\mathbf{r}^{\mathrm{e}}$ siecle inclus, avec une lacune

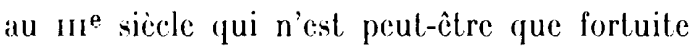
compte tenu du petit nombre d'éléments inventoriés ${ }^{24}$. Il est des lors rertain que l'occupation du site a débulé avant la construction du théatre el qu'elle a perduré au-dela de la ruine de celui-ci.

Ia présence d'un temple est probable. I.e fait que le théàtre ait été bàti sur le plateau, en dépit des commodilés d'aménagement qu'ofiraient les versants d'alentour, semble en effet manifester l'attraction exercée dans cette zone par un édifice public préexistant. Ia topographie des lieux rappelle d'autre part celle des fana de hauteur étudiés par L. de Vesly dans la région rouennaise ${ }^{25}$. Enfin. la proximité de l'éclise paroissiale par rapport

21 I J Mag. 29, 3 J)rag. 37, I Urag. 15/17 011 18/31

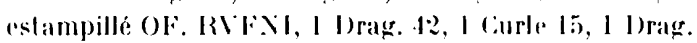
15, 1 Drag. 31, 1 1) rag. 43/Curle 21, 1 1) rag. 90.

25) L.es fana on pelits lemples gallo-romains de la rígion rouennaise, komen, lam. 
au théatre in menviron n'est sans doute pas fortuile. Bien que son vocable. Notre-1)ame. ne présume rien d'ancien. l'existence de la paroisse remonte vraisemblablement à l'époque franque ${ }^{26}$.

I.e territoire de Canouville n'est traversé par aucune voie antique dùment attestée. seul le grand chemin de Fécamp. a $1 \mathrm{~km}$ au nord du théâtre (fig. 1), présente quelques indices d'ancienneté ${ }^{27}$ mais rien ne permet de lui assigner pour autant une origine gallo-romaine.

Il demeure donc beacoup d'incertitudes sur la nalure exacle du site de Canouville. hormis le fait que son développement spatial semble trop limité, trop lacunaire pour qu'il s'agisse d'une ville au plein sens du terme. Gue ce soit un sanctuaire rura ${ }^{28}$, un embryon urbain ${ }^{29}$, ou qu'on le réduise par prudence

26 la nom de danouville vient d'un anthroponyme

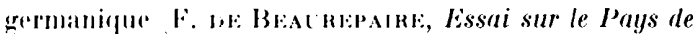
Canx an lemps de la première abbaye de fiécamp, dans Labbaye binfidicline de Fécamp. Congress scienlifique du

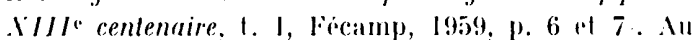
vase funcraire da vo" siecte trouve pres do leaglise cef. ci-dessus, n. 9., il fitut joindre un groupe de sépullures framplues decouvertes en 1966 pres du hameau d'Erneville, a 500 m an sud-est du theâlre Ciallia, X.Vvı, 196x, p. 369 : dessin du mobilier alu C..R....... Lniversite de laten.

27 A peu pres rectiliene sur plusienrs kilomitres, ce chemin se dirige vers saint-Valory-ten-tans ent traversant la valle de Paluel a la hauleur d'un batiment gallo-romain lhbe locin: $\mathrm{T}$, la seine Infórieure historique el archiolongique, Paris, I 666 , p. $45 \mathrm{x}$. II sert de limile infercommunale sur une urande partie de son Irace.

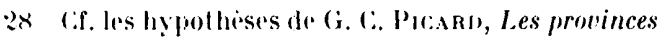
occidentales de l'smpire Romain, dans l.es sources archólugiques de la civilisalion europienne, Bucarest, 1970, p. 15:-16.1.

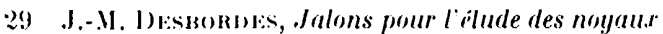
urbains dans lantiquilé gallo-romaine: esemples rígionanx, dans Cahiers archeologiques de Picardie. 197.1, 1. $97-102$.
¿ la simple définition d'“ensemble monumental ${ }^{30}$. sa découverle n'est rependant pas sans ronséquences. $14 \mathrm{~km}$ au sud-est de Canouville, au fond de la vallée de la IJurdent, se trouve un bourg du nom de Cany qui passe pour avoir joué le role d'une sorte de petit chef-lieu régrional à l'époque gallo-romaine ${ }^{31}$. Il conviendra désormais de considérer que nous ne sommes plus en présence d'une agrolomération unique, mais de deux sites a vocations: différentes et semble-l-il complementaires : une bourgade routiere el commerciale dans la vallée, sur le passage d'eau. un lieu de rassemblement public sur la hauteur, à la lisiere des bois. Il faudrail faire enfin la part des antécédents préromains. L'oppidum maritime de Veuletles. a $\vdots \mathrm{km}$ de Canourille. sugriere l'existence d'une slation indigine entretenant des rapporls commerciaux avec l'île de Brelagne ${ }^{32}$.

Jacques La Mano et Jean-Marie Vicon.l.:.

30 F. I) Inasv. Les thitres ruraur des carmutes ol des senons. Leur implantalion et lears rapports anec la civilas, dans Rerue archéologique du cientre, 13, fasc. 3-1, p. 195 et $\mathrm{s}$

31 Ahbe Cor:n1: I.n Seine Inferienre hislorique el archeologique, l'aris, I 666, p. 14x-152. Des observalions phus riocentes monternt que le vicus s'olendail vers le sud jusqua l'emplacement du terain de sporl de dany, el vers le nord juşüa la hauleur du chàleau de laniel

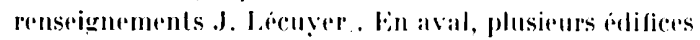
isolis onl ble recommus sur une distance de $4 \mathrm{~km}$

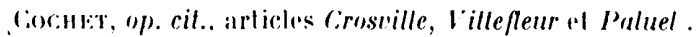

32 .I. Whisis, K. Rurnanusox, Hill forls of Sorthern France, (1xford, 195\%, 1). 123. Sur un depol de bronze tromve an Hanouard, pros do lany, ef. (i. Vвниох, Musée déparlemental des antiquiles de la seine-Warilime. Anliquiles prohistoriques el prolohisloriques, Ihouren, 1971, p. 56 at 57.

X.B. . I I cliche de la lig. 1 est dì à M. I.ecuser. 